\title{
Rückzug chinesischer Arzneimittel mit toxischen Stoffen
}

Swissmedic

\begin{abstract}
Die Methoden der traditionellen chinesischen Medizin (TCM) werden in der Schweiz immer häufiger eingesetzt. Bestandteil der TCM sind auch Arzneimittel aus Pflanzen, Mineralien und teilweise tierischem Material. In der Schweiz sind davon mehrere Hundert im Handel. Sie werden in der Regel auf individuelle Rezeptur eines Arztes verschrieben oder auf Empfehlung eines Therapeuten abgegeben.
\end{abstract}

Tabelle 1

In der TCM gebräuchliche Arzneipflanzen, welche toxische PA enthalten.

\begin{tabular}{lll} 
pin Yin & pin Yin Synonym & Botanischer Name \\
\hline Zi Cao & Zi Cao Gen & Arnebia euchroma (Royle) Johnst \\
\hline Zi Cao & Zi Cao Gen & Arnebia guttata Bunge \\
\hline Zi Cao & Zi Cao Gen & Lithospermum erythrorhizon Sieb. et Zucc. \\
\hline Zi Cao & Zi Cao Gen & Lithospermum euchroma Royle \\
\hline Kuan Dong Hua & & Tussilago farfara L. \\
\hline Jiu Li Ming & Qian Li Guang & Senecio scandens Buch-Ham. \\
\hline Pei Lan & & Eupatorium fortunei Turcz. \\
Pei Lan & & Eupatorium shimadai Kitamura \\
Tu San Qi & & Senecionis chrysanthemoidis
\end{tabular}

Korrespondenz:

Swissmedic

Erlachstrasse 8

CH-3000 Bern 9

Tel. 0313220211

Fax 0313220212
In einigen dieser Arzneimittel kommen Pflanzen vor, die sogenannte Pyrrolizidinalkaloide (PA) enthalten. Diese Alkaloide sind in höheren Konzentrationen potenziell leberschädigend und bei chronischer Anwendung können sie Krebs auslösen. In der Schweiz und in anderen europäischen Ländern gibt es deshalb seit über 10 Jahren Grenzwerte für diese Inhaltsstoffe. Swissmedic hat mehrere Firmen von TCM-Arzneimitteln, welche solche Pflanzenzubereitungen in ihrem Sortiment führen, aufgefordert, Angaben zur PA-Konzentration ihrer Präparate zu liefern. Die heute vorliegenden Daten zeigen, dass neben Produkten, die unter dem Grenzwert liegen, mehrere Arzneimittel den erlaubten Grenzwert um ein Vielfaches überschreiten. Die entsprechenden Präparate wurden deshalb von Swissmedic für die Abgabe gesperrt und von den verantwortlichen Vertriebsfirmen vom Markt zurückgerufen. Vom Rückruf betroffen waren bisher einzelne Chargen folgender Pflanzen: Pei Lan: Eupatorii herba; Qian Li Guang: Senecionis scandentis herba; Kuan Dong Hua: Farfarae flos sowie Zi Cao: Lithospermi radix.

Da fast alle entsprechenden Präparate aus dem Fernen Osten importiert werden, hat Swissmedic auch die ausländischen Arzneimittelbehörden über diese Ergebnisse informiert und ihnen empfohlen, bei Arzneipflanzen, welche toxische PA enthalten könnten, von den Vertriebsfirmen routinemässig eine Gehaltsprüfung $\mathrm{zu}$ verlangen.

Auch Swissmedic erhält von Arzneimittelbehörden aus der ganzen Welt regelmässig Warnmeldungen über verunreinigte oder absichtlich verfälschte TCM-Arzneimittel. Allein letzte Woche gingen zwei solcher Meldungen ein. Sie betrafen chinesische Schlankheitsmittel (Produktbezeichnungen: Qing Zhisan Tian Shou, Kapseln, und Lida DaiDaihua), die als rein pflanzlich deklariert waren, obwohl sie den stark wirksamen synthetischen Wirkstoff Sibutramin enthielten. Häufig ist wegen ungenügender Beschriftung der Produkte nicht klar, von welchem Hersteller solche Präparate letztlich stammen und ob sie einer Qualitätskontrolle unterliegen. Entsprechende Arzneimittel werden leider auch von Patienten häufig (z. B. via Bestellung per Internet) direkt aus dem Ausland bezogen. Solche Sendungen können die Gesundheit gefährden, weil oft keine oder nur ungenügende Angaben zur Qualität vorliegen und auch keine Gewähr besteht, dass das Arzneimittel wirklich jene Wirkstoffe enthält, die auf der Packung deklariert sind. Es wird deshalb empfohlen, Arzneimittel der TCM nur von Firmen zu beziehen, die in der Schweiz zum Vertrieb solcher Präparate autorisiert sind. Diese sind zu regelmässigen Qualitätskontrollen verpflichtet. 


\section{Retrait de médicaments chinois contenant des substances toxiques}

Swissmedic

\begin{abstract}
La médecine traditionnelle chinoise (MTC) gagne en popularité en Suisse. Certains produits utilisés par cette médecine sont des médicaments à base de plantes, de minéraux et pour certains de substances d'origine animale. On en trouve plusieurs centaines dans le commerce en Suisse. Ces médicaments sont généralement prescrits individuellement par un médecin ou remis sur recommandation d'un thérapeute.
\end{abstract}

Tableau 1

Drogues végétales contenant des AP et usuelles en MTC.

\begin{tabular}{lll} 
pin Yin & synonyme pin Yin & nom botanique \\
\hline Zi Cao & Zi Cao Gen & Arnebia euchroma (Royle) Johnst \\
\hline Zi Cao & Zi Cao Gen & Arnebia guttata Bunge \\
\hline Zi Cao & Zi Cao Gen & Lithospermum erythrorhizon Sieb. et Zucc. \\
\hline Zi Cao & Zi Cao Gen & Lithospermum euchroma Royle \\
\hline Kuan Dong Hua & & Tussilago farfara L. \\
\hline Jiu Li Ming & Qian Li Guang & Senecio scandens Buch-Ham. \\
\hline Pei Lan & & Eupatorium fortunei Turcz. \\
Pei Lan & & Eupatorium shimadai Kitamura \\
Tu San Qi & & Senecionis chrysanthemoidis
\end{tabular}

Korrespondenz:

Swissmedic

Erlachstrasse 8

CH-3000 Berne 9

Tél. 0313220211

Fax 0313220212
Certains de ces médicaments sont issus de plantes contenant un alcaloïde de pyrrolizidine $(\mathrm{AP})$, qui peut être hépatotoxique à hautes concentrations, voire provoquer un cancer lors d'une utilisation de longue durée. C'est pourquoi la Suisse et d'autres pays européens ont fixé il y a plus de dix ans déjà des valeurs limites pour ces composants toxiques. Swissmedic a enjoint plusieurs entreprises qui proposent des préparations à base de telles plantes de lui fournir des données sur la concentration en AP de leurs préparations. Les données disponibles aujourd'hui montrent que, si certains produits affichent une valeur inférieure à la limite fixée, d'autres par contre la dépassent de beaucoup. Swissmedic a donc interdit la remise de ces produits, qui ont été retirés du marché par les distributeurs respon- sables. Voici les plantes dont des lots ont été retirés du marché: Pei Lan: Eupatorii herba; Qian Li Guang: Senecionis scandentis herba; Kuan Dong Hua: Farfarae flos ainsi que Zi Cao: Lithospermi radix.

$\mathrm{Du}$ fait que la plupart des préparations concernées sont importées d'Extrême-Orient, Swissmedic a communiqué les données aux autorités étrangères de contrôle des médicaments, en leur recommandant d'exiger des distributeurs de drogues végétales susceptibles de contenir des AP de leur soumettre à titre routinier des analyses de la teneur en AP.

Soulignons que Swissmedic reçoit régulièrement des autorités de contrôle des médicaments du monde entier des alertes sur des médicaments de la MTC contaminés ou délibérément contrefaits. Ainsi, la semaine passée, deux annonces de ce type ont été saisies, qui portaient sur des produits amaigrissants chinois déclarés comme purement végétaux (vendus sous la dénomination: Qing Zhisan Tian Shou, capsules et Lida DaiDaihua), alors qu'ils contenaient de la sibutramine, principe actif synthétique très puissant. Souvent, les inscriptions sur les emballages sont peu lisibles et il est donc difficile de trouver le nom du fabricant responsable des produits et de vérifier si ceux-ci ont fait l'objet d'un contrôle de la qualité. Malheureusement, nombreux sont ceux qui achètent de tels produits directement à l'étranger, par exemple en les commandant par Internet. Or, ces produits peuvent présenter un risque pour la santé, d'autant plus qu'ils ne comportent pas assez d'informations, voire aucune, sur la qualité et que rien ne permet donc d'assurer qu'ils comprennent effectivement les principes actifs déclarés sur l'emballage. Il est donc vivement conseillé de n'acheter des médicaments de la MTC que d'entreprises qui possèdent une autorisation de distribuer de tels produits en Suisse et qui, de ce fait, sont soumises régulièrement à des contrôles de la qualité. 